
\section{E

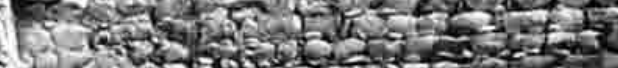 El fluoruro de sodio, una alternativa para la conservación
de roca caliza disgregada} $=$

\author{
uisa Straulino
}

- n la época prehispánica, la roca fue un material ampliamente utilizado - para la construcción de edificios o para la talla de diferentes elementos decorativos. Por eso el estudio de los materiales pétreos, el análisis de su intemperismo y la búsqueda de soluciones para disminuir su deterioro es de suma importancia para su preservación hacia el futuro.

Los mayas tenían una gran disponibilidad de materiales pétreos, ya que se asentaron en una plataforma de roca caliza $\left(\mathrm{CaCO}_{3}\right)$. En particular, la región Río Bec que se encuentra al sureste del estado de Campeche está asentada en las formaciones geológicas calizas denominadas Xpujil (Tpe) e Icaiché (Te). ${ }^{1}$ Así, los edificios que aún permanecen en el sitio arqueológico de Río Bec están construidos y decorados con roca caliza y otros derivados como la cal, polvo de piedra, sascab ${ }^{2}$, etcétera (García Solís et al. 1997), que se obtenían de canteras locales (Arnauld et al. 2008).

Estos edificios presentan una problemática de conservación compleja, ya que su estado de conservación se ha agravado debido a la disgregación de la roca caliza constitutiva (Michelet et al. 2007, 2008 y 2009). Además, diversas fuentes de información señalan que esta problemática se extiende a las zonas arqueológicas aledañas que forman parte de la región estilística de Río Bec, como en el caso de Chicanná, Kohunlich y Dzibanche, ${ }^{3}$ donde, desde hace varios años, se han llevado a cabo diversas investigaciones en materia de conservación arqueológica (García Solís y Valencia 1996; García et al. 1996 y 1997; Anrubio 1998).

Se ha reportado que el principal problema de conservación es la formación de sulfatos provenientes de la composición de la roca. Sin embargo, esta afir-

${ }^{1}$ La formación Xpujil está integrada por secuencias de caliza microcristalina, de textura mudstone, estratificada, con cambios de facies hacia limos y lutitas, así como depósitos de anhidrita y yeso con horizontes arcillosos escasos e intercalaciones de caliza hacia la parte superior de la unidad. La formación Icaiché está integrada por una secuencia de caliza estratificada y ocasionalmente masiva, de textura mudstone con raros fragmentos de sílice coloidal, y algunos cambios de facies hacia limos y arcillas; presenta también halita en su composición (Castro 2002; Conagua 2007).

2 Sascab (traducido del maya yucateco como "tierra blanca") es un agregado mineral comúnmente utilizado en los morteros de cal en el área maya. Proviene de sustratos de caliza no consolidados.

${ }^{3}$ Chicanná es una zona arqueológica perteneciente a la región Río Beck, Kohunlich y Dzibanché, aunque no son parte de esta región, tienen elementos estilísticos de esta tradición desarrollados en ciertos periodos de su desarrollo y están relativamente cerca del sitio. 
mación no ha sido comprobada de manera científica. De hecho, las causas y mecanismos de alteración de las rocas calizas en esta región maya no son bien conocidos, por lo que es necesario analizar los propios procesos de deterioro de las rocas calizas de la región de Río Bec, para poder proponer una solución viable para su conservación.

En las últimas décadas, se han utilizado diversos procesos para consolidar las rocas calizas disgregadas. Los polímeros sintéticos fueron descartados en la década de los noventa debido a sus efectos contraproducentes (Cedillo 1991; Castro y Tapia 1993 ). Actualmente se prefiere el uso de sustancias inorgánicas compatibles con el material original, tales como el hidróxido de bario, formiato de bario, silicatos de etilo e hidróxido de calcio. ${ }^{4}$ Este último compuesto, en su presentación de agua de cal, se ha utilizado con una mayor amplitud. No obstante, su aplicación requiere una gran inversión de recursos, debido a que un litro de solución acuosa contiene aproximadamente $1.7 \mathrm{~g}$ de materia sólida, que al reaccionar con el anhídrido carbónico ambiental, formará cerca de $2.3 \mathrm{~g}$ de carbonato de calcio. Por tanto, se requiere de un gran número de aplicaciones para obtener un efecto consolidante apreciable en la resistencia de la roca (Ashurst y Dimes 1998: 166).

Respecto a lo anterior, Luis Torres Montes $(1967)^{5}$ señala que para consolidar piedra caliza, el agua de cal debe aplicarse de 30 a 40 veces. Por otro lado, en la literatura extranjera se proponen variantes al procedimiento de la simple aplicación de agua de cal por aspersión. En la catedral de Walles (Caroe 1986:106) cada elemento restaurado fue consolidado mediante la aplicación de un emplasto de cal hidratada que se mantuvo húmeda durante tres semanas. Después el emplasto fue removido y por último fueron necesarias de 30 a 50 aplicaciones de agua de cal para obtener un resultado adecuado en términos de cohesión. Este proceso es muy similar al utilizado para la aplicación de hidróxido de bario (Lewin 1974: 25).

La consolidación con agua de cal conlleva otros problemas, ya que aporta cantidades elevadas de agua, lo que puede acelerar el deterioro por cristalización de sales solubles o disgregación de materiales arcillosos presentes en la composición de la roca.

Tomando en cuenta lo anterior, el tratamiento de roca caliza disgregada requiere del desarrollo de nuevas alternativas que, de manera sencilla y rápida, propicien una mayor estabilidad en las rocas que conforman la arquitectura prehispánica ubicada en zonas tropicales húmedas.

\footnotetext{
${ }^{4}$ Recientemente en Europa se han utilizado nanopartículas de hidróxido de calcio o bario como consolidante de roca (Giorgio Torraca, comunicación personal, 2009) . En México, no se han encontrado reportes de su utilización como tal, aunque sí para la pintura mural. Por ejemplo, se han utilizado en Calkmul (restauradora María Masaguer, comunicación personal, 2009), y se utilizaron también en la Caja de Agua de Tlatelolco, entre otros sitios con pintura mural, por citar algunos casos.

${ }^{5}$ Citado en la tesis “La conservación en zonas arqueológicas. Tres décadas de trabajo" (Cedillo 1991: 93).
}

En este trabajo se propone, como alternativa de tratamiento, la remineralización con fluoruro de sodio $(\mathrm{NaF}){ }^{6}$ el cual al interactuar con el carbonato de calcio $\left(\mathrm{CaCO}_{3}\right)$ de la roca caliza, forma fluoruro de calcio $\left(\mathrm{CaF}_{2}\right)$. Este cambio en la composición mejora las cualidades físicas de las rocas calizas, y además provee de una capa insoluble a la superficie tratada. ${ }^{7}$

Para el estudio se utilizaron muestras provenientes de una cornisa decorativa conformada por 77 piezas del edificio 5N2 de Río Bec. Estas piezas fueron encontradas durante las excavaciones de las temporadas tres, cuatro y cinco (años 2004, 2005 y 2006) del Proyecto Arqueológico Río Bec, ${ }^{8}$ en la fachada norte del Edificio 5 N2 del Grupo A; justo en el nivel de destrucción del edificio, bajo una capa de suelo enriquecida en humus (Michelet et al., 2005: OpVA/3) (Figura 1). Además se utilizaron algunas muestras tratadas e intemperizadas in situ que provienen de la fachada Este del edificio 6N2.

\section{Metodología}

Los análisis empleados para conocer la composición específica de la roca, los agentes de intemperismo, sus efectos en el material pétreo, así como los cambios provocados por la aplicación de fluoruro de sodio como remineralizador fueron divididos en dos secciones: las técnicas encaminadas a conocer la composición química y mineralógica de las muestras tanto remineralizadas como sin remineralizar; y los métodos utilizados para conocer las propiedades físicas de las muestras tanto remineralizadas como sin remineralizar. A continuación se detallan dichos análisis.

Para conocer la composición mineralógica y química se usó: microscopía petrográfica, ${ }^{9}$ microscopía electrónica de barrido (MEB) con microsonda (EDS) ${ }^{10}$ y difracción de rayos $X(D R X){ }^{11}$

Para conocer las propiedades físicas se realizaron mediciones de: densidad real, ${ }^{12}$ densidad aparente, ${ }^{13}$ porosi-

\footnotetext{
${ }^{6}$ La única contraindicación del fluoruro de sodio es que no se puede aplicar con herramientas que tengan metal o vidrio, ya que el flúor contenido en el compuesto, se precipita y el producto ya no funciona.

${ }^{7}$ Parte importante del deterioro de las rocas calizas es causado por su alta solubilidad en agua ácida. En condiciones normales el agua de la superficie terrestre y de la lluvia es ácida, por la disolución de $\mathrm{CO}_{2}$ generando cantidades variables de ácido carbónico.

${ }^{8}$ El proyecto Río Bec: Sociedad y economía de Río Bec en su apogeo, está dirigido por el doctor Dominique Michelet, la doctora Charlotte Arnauld y el doctor Philippe Nondédéo.

9 Láminas delgadas, técnico Eligio López. Asesoría y fotografía doctor Sergey Sedov, Instituto de Geología, Unam.

10 Fotografías: doctora Silvia Antuna, Facultad de Medicina, UNAM; doctora Yolanda Ornelas, Instituto de Ciencias del Mar y Limnología; maestro Gerardo Villa, Subdirección de Laboratorios y apoyo académico del INAH, EDS; maestro Gerardo Villa, Subdirección de Laboratorios y apoyo académico del INAH.

11 Doctora Teresa Pi Puig, Instituto de Geología, UnAm.

12 Asesoría René Alcalá, Instituto de Geología, UNAM.

${ }^{13}$ Asesoría René Alcalá, Instituto de Geología, unam.
} 


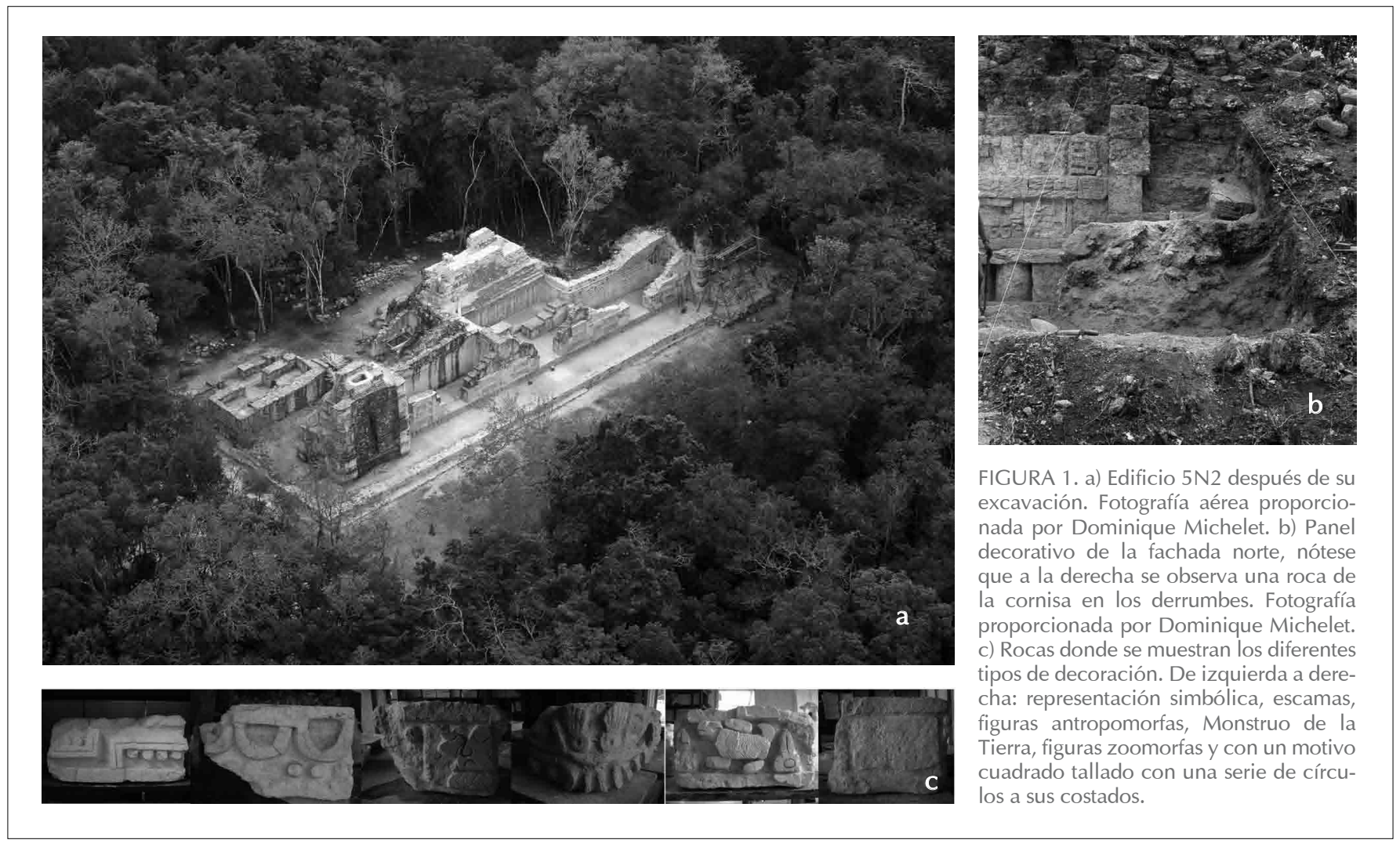

dad, ${ }^{14}$ retención de humedad, ${ }^{15}$ colorimetría ${ }^{16}$ y dureza Shore D. ${ }^{17}$

Los análisis anteriores proporcionaron datos suficientes para evaluar el tratamiento de conservación de roca caliza por medio del fluoruro de sodio.

La extracción de las muestras de roca-tomadas in situ, antes de aplicar cualquier tratamiento- se realizó de dos maneras: recolección del material de deshecho de las rocas de la cornisa, es decir, fragmentos sueltos de piedra disgregada, para no afectar más la estructura de los elementos constructivos originales; y extracción mediante cortes con objetos punzocortantes en rocas sin deterioro con disgregación moderada. Cabe mencionar que aunque el tamaño de las muestras fue heterogéneo el promedio fue de $2 \mathrm{~cm}^{3}$ por ejemplar.

Para remineralizar las muestras con fluoruro de sodio (NaF), se utilizó el método de inmersión, ya que éste asegura la mayor penetración posible sin utilizar bombas de vacío. Cada muestra se sumergió en $80 \mathrm{ml}$ de una solución de $\mathrm{NaF}$ a $1 \%$ en agua destilada, durante 24 horas $^{18}$ para su remineralización.

\footnotetext{
14 Asesoría René Alcalá, Insituto de Geología, UnAM.

15 Diseño experimental: doctor Jorge Gama Castro, maestro Jaime López (Instituto de Geología, UNAM) y Luisa Straulino (enCRym, INAH).

${ }^{16}$ Maestro Manlio Favio Salinas, ENCRYM, INAH.

17 Doctor Alfredo Maciel, Instituto de Investigación en Materiales, UNAM.

18 Se escogió una solución a $1 \%$ ya que soluciones a mayor concentración generan tanta dureza en el material que se quiebra fácilmente (Luisa María Mainou, comunicación personal 2009). Para comprobarlo se realizaron probetas a $1 \%, 2 \%, 4 \%, 5 \%$ y $6 \%$, a partir de $2 \%$ todas las
}

También se llevaron a cabo pruebas de remineralización por aspersión, con el objetivo de comparar el grado de penetración de un método alternativo de aplicación del producto, usado comúnmente en las intervenciones de grandes áreas por su facilidad y rapidez, aspecto fundamental para su posterior aplicación en zonas arqueológicas. Se llevaron a cabo 10 aspersiones con la misma solución a $1 \%$ de $\mathrm{NaF}$ en agua destilada.

Por último, el producto se aplicó con brocha en la mitad de una piedra caliza -en una zona libre de tratamientos de restauración, según el doctor Dominique Michelet director del Proyecto Río Bec- perteneciente al edifico 6N2 de Río Bec. Al transcurrir de un año se tomaron muestras de la zona remineralizada y no remineralizada, para realizar los análisis correspondientes.

A continuación se presenta el número de muestras utilizadas para cada estudio (Tabla 1).

\section{Resultados}

Composición: las rocas calizas pertenecientes a la cornisa del edificio 5N2 de Río Bec están formadas principalmente por micrita, además se pueden encontrar rasgos primarios como estructuras fluidales, organismos que han sido sustituidos por calcita, oolitos; así como rasgos diagenéticos como la formación de esparita y desdolomitización.

rocas se encontraron muy quebradizas. Por otro lado se aplicó el fluoruro de sodio con herramientas sin metal, ya que cuando la solución entra en contacto con algún metal el flúor se precipita consumiendo los iones que reaccionarán con el calcio. 


\begin{tabular}{|c|c|c|c|}
\hline Análisis & $\begin{array}{l}\text { Número de } \\
\text { rocas utilizadas }\end{array}$ & $\begin{array}{l}\text { Número de } \\
\text { muestras utilizadas }\end{array}$ & Observaciones \\
\hline Petrografía & 8 & 16 & $\begin{array}{l}\text { El número de muestra se duplica con respecto a las rocas } \\
\text { usadas, ya que se analizó una muestra de roca sin trata- } \\
\text { miento y otra remineralizada. }\end{array}$ \\
\hline $\begin{array}{l}\text { Micromorfo- } \\
\text { logía en MEB }\end{array}$ & 4 & 8 & $\begin{array}{l}\text { El número de muestra se duplica con respecto a las rocas } \\
\text { usadas, ya que se analizó una muestra de roca sin trata- } \\
\text { miento y otra remineralizada. }\end{array}$ \\
\hline $\begin{array}{l}\text { Composición } \\
\text { química EDS } \\
\text { (espectrogra- } \\
\text { mas y mapeos) }\end{array}$ & 8 & 13 & $\begin{array}{l}\text { De las ocho rocas utilizadas, cinco fueron muestreadas } \\
\text { para analizarlas sin tratamiento y remineralizadas por in- } \\
\text { mersión dando un total de } 10 \text { muestras; y, las otras tres } \\
\text { fueron muestreadas para ser remineralizadas por aspersión } \\
\text { para comparar la penetración por método de aplicación. }\end{array}$ \\
\hline $\begin{array}{l}\text { Composición } \\
\text { mineralógica } \\
\text { DRX }\end{array}$ & 4 & 8 & $\begin{array}{l}\text { El número de muestra se duplica con respecto a las rocas } \\
\text { usadas, ya que se analizó una muestra sin tratamiento y } \\
\text { otra remineralizada. }\end{array}$ \\
\hline Densidad real & 4 & 8 & $\begin{array}{l}\text { El número de muestra se duplica con respecto a las rocas } \\
\text { usadas, ya que se analizó una muestra sin tratamiento y } \\
\text { otra remineralizada. }\end{array}$ \\
\hline $\begin{array}{l}\text { De n s id a d } \\
\text { aparente }\end{array}$ & 4 & 38 & $\begin{array}{l}\text { El experimento se tuvo que repetir varias veces con cada } \\
\text { roca para obtener un mejor resultado promediando los } \\
\text { valores obtenidos. }\end{array}$ \\
\hline Porosidad & 0 & 0 & Se calcula con los dos valores anteriores. \\
\hline $\begin{array}{l}\text { Retención de } \\
\text { humedad }\end{array}$ & 3 & 30 & $\begin{array}{l}\text { Se hicieron repeticiones de cada roca para obtener un } \\
\text { promedio. }\end{array}$ \\
\hline Colorimetría & 5 & 10 & $\begin{array}{l}\text { El número de muestras se duplica con respecto a las rocas } \\
\text { usadas ya que se analizó una muestra sin tratamiento y } \\
\text { otra remineralizada. }\end{array}$ \\
\hline Dureza & 4 & 8 & $\begin{array}{l}\text { El número de muestras se duplica con respecto a las rocas } \\
\text { usadas ya que se analizó una muestra sin tratamiento y } \\
\text { otra remineralizada. }\end{array}$ \\
\hline
\end{tabular}


Además de la calcita, que es el mineral principal, se encuentran otros minerales en mucho menores cantidades como pedernal, cuarzo, sílice, halita y arcillas esmectíticas.

Esto coincide con la composición de las rocas de las formaciones Icaiché y Xpuhil.

Deterioro o intemperismo: los rasgos de intemperismo observados en las muestras, por medio de láminas petrográficas y observación en MEB, fueron diversos. Se identificó con mucha frecuencia la falta de cementación entre granos individuales de micrita y la formación de carbonatos secundarios en forma de aguja (cristales aciculares). Estos últimos se observaron en la superficie de algunas
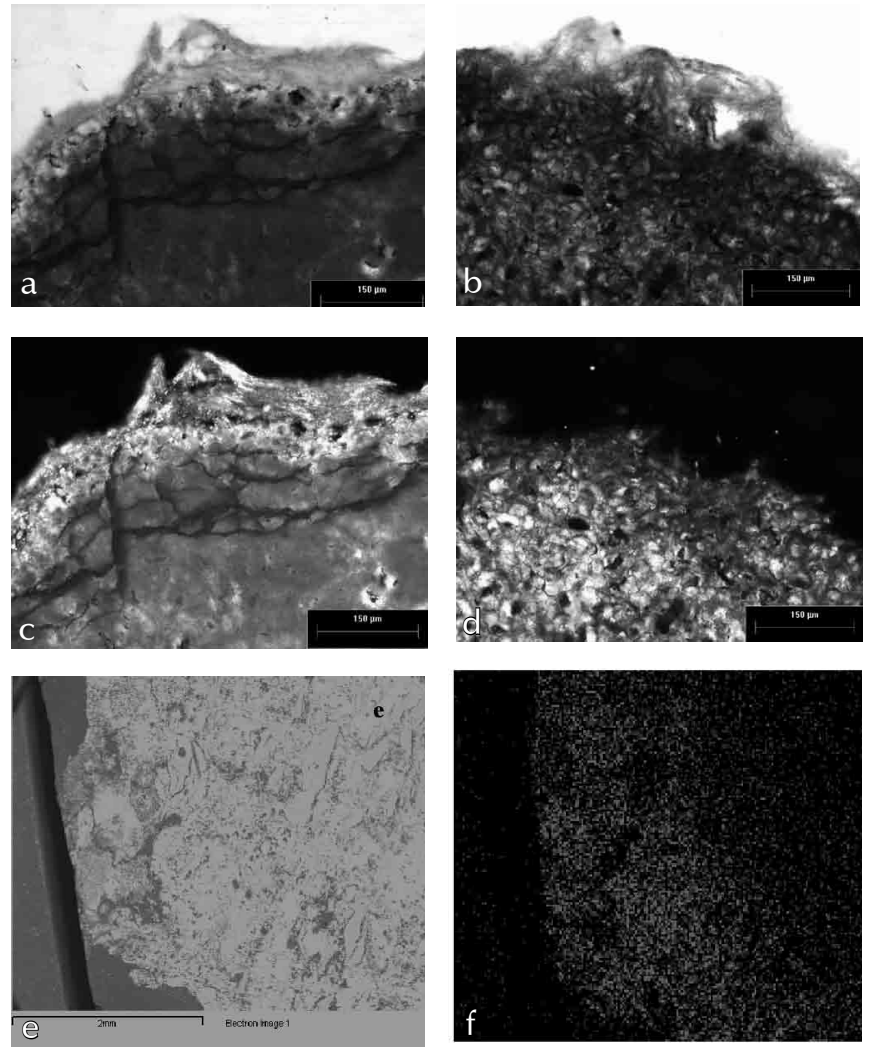

F Ka1_2

FIGURA 2. Se muestra el caso de la roca T22.5.

a) Superficie sin tratamiento que presenta cristales aciculares. Luz transmitida 10x.

b) Superficie de la misma roca pero remineralizada. También presenta cristales aciculares. Compárese el color de la superficie con la Figura 2a, en esta fotografía el color es más oscuro. Luz transmitida 10x. c) Superficie de roca sin tratamiento con cristales acicualres. Nicoles cruzados 10x

d) Superficie de roca remineralizada con cristales aciculares. Nicoles cruzados 10x. Nótese que los cristales aciculares no tienen colores de interferencia altos, además la micrita superficial también se observa oscura.

e) Corte transversal de roca remineralizada observada en microscopio de barrido con EDS.

f) Mapeo del elemento flúor, se observa que se distribuye en una banda en la superficie. de las muestras -las que presentaban un mayor grado de disgregación- y en la mayoría de los poros de las rocas.

Existen dos causas de la formación de estos cristales: la disolución y posterior recristalización de calcita en presencia de hifas fúngicas; $y$, la disolución y posterior recristalización en presencia de raíces. Cada causa de deterioro provoca formaciones distintas de cristales aciculares. Los cristales que no presentan un orden específico, encontrados en poros y superficie de las muestras, tienen relación con hifas fúngicas.

Por un lado, los canales formados por microrraicillas que penetraron la estructura de la roca disolviéndola, tienen una micromorfología particular. Cada canal esta formado por diversas celdas -tipo panal- que parecen copiar en un negativo la superficie de las raíces que les dieron origen. Conjuntamente cada celda está formada por cristales aciculares de distintos tamaños, entramados finamente.

Por el otro lado, se observó que la superficie de las rocas intemperizadas, se encuentra pulverulenta, ya que tiene granos de micrita poco cementados al núcleo de la roca. El grado de cementación es inversamente proporcional al grado de disgregación de la roca. Además, se pueden observar patrones de intemperismo debidos a la oxido-reducción de iones férricos.

Comparación de rocas remineralizadas y no remineralizadas: durante la observación de las muestras remineralizadas y sin tratamiento, se notó -mediante las distintas técnicas microscópicas- la formación de una zona oscura en la superficie de las muestras remineralizadas. Esto indicaba la posible conversión de calcita a fluorita por varias razones: a) el fluoruro de calcio tiene un índice de refracción mucho menor al de la calcita y presenta un alto relieve óptico. Estas características de la fluorita generan que se observe más oscura que la calcita cuando es examinada con luz transmitida; b) la fluorita cristaliza en un sistema cúbico, por lo que es isotrópica. Por ello cuando se observan las muestras remineralizadas con nicoles cruzados, la fluorita no posee colores de interferencia y, por lo tanto, se observa un área oscura; esto contrastará fuertemente con la calcita que, por tener sistemas cristalinos anisotrópicos, presenta altos colores de interferencia; $c$ ) cuando las muestras son observadas en MEB, los colores más oscuros se encuentran donde hay elementos más ligeros, por lo tanto la fluorita se observó más oscura que la calcita (Figura 2).

Los cristales de las rocas remineralizadas y sin tratamiento mantienen el mismo hábito cristalino por un tiempo indeterminado, antes de un año de haber aplicado el tratamiento (forma de micrita y cristales aciculares). Se comprobó que un año después de su aplicación los cristales desarrollan un hábito cristalino similar al de la fluorita (cristales aciculares formados por subunidades cúbicas) (Figura 3).

Después de obtener estos resultados, las probetas se analizaron con DRX, comprobando que la reminerali- 


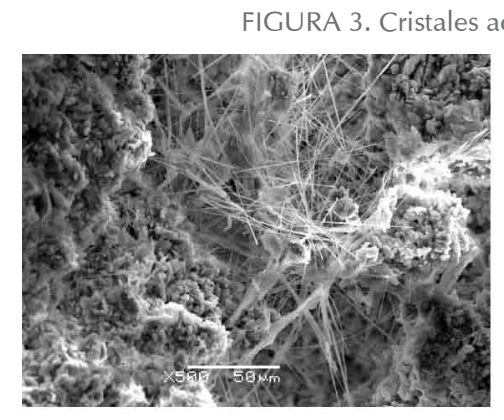

a) Cristales aciculares de roca sin tratamiento.

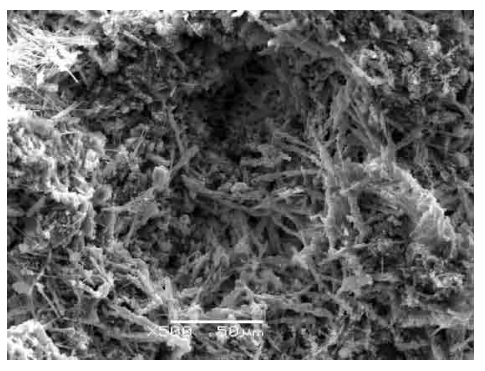

b) Critales aciculares de roca tratada.

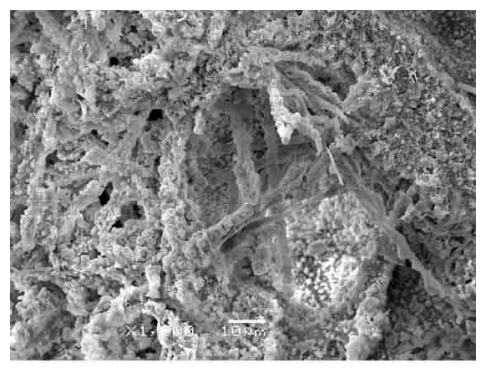

c) Cristales aciculares de roca remineralizada e intemperizada durante un año.

zación convierte al carbonato de calcio en fluoruro de calcio. Entonces se observó que las rocas no tratadas presentaban carbonato de calcio, arcillas y cuarzo en su composición; mientras que las remineralizadas contenían fluoruro de calcio, además de los minerales antes mencionados (Figura 4).

Mediante mapeo elemental en cortes transversales de las rocas se determinó que el flúor penetra en la superficie de 64 micras a $2 \mathrm{~mm} .{ }^{19}$ La capacidad de penetración del flúor está determinada por la conformación de la red porosa de la roca, así como por el método de aplicación.

Por otro lado, se verificaron algunos cambios en las propiedades físicas, a saber: la densidad real, como se puede observar en la Tabla 2 es menor en las rocas tratadas, puesto que los átomos se desacomodan en las celdas unitarias de los cristales cuando se lleva a cabo el intercambio entre el ion carbonato y el ion flúor.

La densidad aparente (Tabla 3) en las rocas tratadas es menor que en la rocas no tratadas. Esto podría atribuirse a que los poros se limpian de impurezas, material soluble y material disgregado, durante el tratamiento de remineralización.

La porosidad de las rocas remineralizadas es mayor que la de las rocas no tratadas (Tabla 4). Este cambio en la porosidad tiene como consecuencia varias transformaciones en comparación con las rocas no tratadas: a) la densidad aparente de las rocas remineralizadas disminuye; b) las rocas tratadas absorben más agua que las no tratadas. Sin embargo, en las rocas remineralizadas, el secado es más rápido y la cantidad de agua que se evapora es mayor, lo cual implica que el agua tiene menos tiempo de actuar en la formación y transportación de sales dentro de la estructura de la roca, además las sales cristalizarán como eflorescencias y no como subeflorescencias.

Al analizar los resultados obtenidos mediante colorimetría se determinó que no existe un cambio importante en el color de las muestras remineralizadas en comparación con las rocas sin tratamiento; de los tres parámetros registrados (saturación, tono y luminosidad) únicamente aumenta la saturación.

${ }^{19}$ La recesión anual de la roca caliza se estima entre 4 y 10 micras. Por lo tanto la recesión anual de una roca cuya superficie se ha transformado en fluoruro de calcio virtualmente insoluble en agua, será mucho menor. Una capa de 64 micras tardará más de 6 años (tomando como referencia la recesión mayor reportada para calcita) en disolverse. $\mathrm{H}$. Bravo-Álvarez et al. (2003).

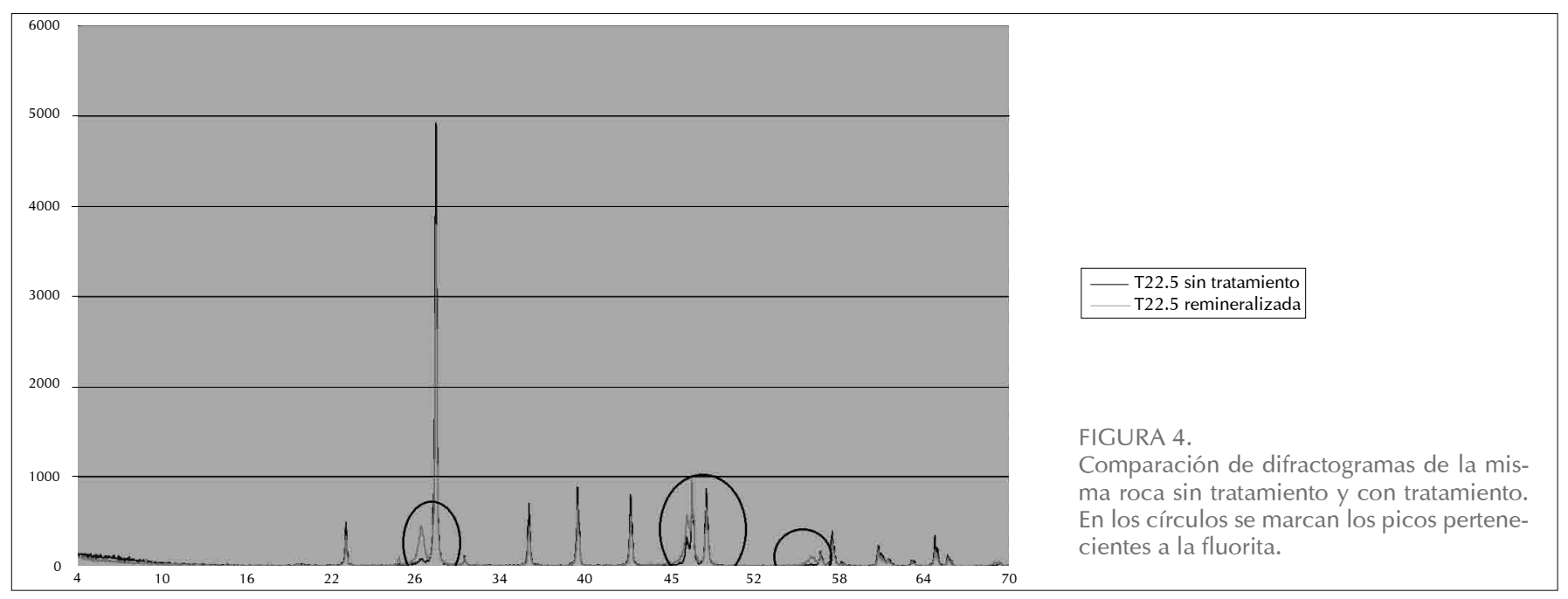




\begin{tabular}{|c|c|c|c|c|}
\hline $\begin{array}{c}\text { Clasificación de } \\
\text { las rocas }\end{array}$ & $\begin{array}{c}\text { Sin } \\
\text { remineralizar } \\
(\mathrm{g} / \mathrm{cm} 3)\end{array}$ & $\begin{array}{c}\text { Remineralizadas } \\
(\mathrm{g} / \mathrm{cm} 3)\end{array}$ & Diferencia & Porcentaje \\
\hline $\mathrm{T} 22.5$ & 2.98 & 2.957 & 0.023 & $1.14 \%$ \\
\hline $\mathrm{T} 14.3$ & 2.907 & 2.874 & 0.033 & $1.46 \%$ \\
\hline $\mathrm{T} 21.4$ & 2.807 & 2.766 & 0.041 & $2.79 \%$ \\
\hline $6 \mathrm{~N} 2$ & 2.654 & 2.728 & 0.074 & 2 \\
\hline
\end{tabular}

\section{Tabla 3. Resultados promedio de densidad aparente}

\begin{tabular}{|c|c|c|}
\hline Roca & $\begin{array}{c}\text { Sin } \\
\text { remineralizar } \\
\text { (g/cm3) }\end{array}$ & $\begin{array}{c}\text { Remineralizada } \\
\text { (g/cm3) }\end{array}$ \\
\hline $\mathrm{T} 14.3$ & 2.327 & 2.388 \\
\hline $\mathrm{T} 22.5$ & 2.186 & 2.029 \\
\hline $\mathrm{T} 21.4$ & 1.96 & 1.895 \\
\hline $6 \mathrm{~N} 2$ & 2.019 & 1.8875 \\
\hline
\end{tabular}

Tabla 4. Resultados promedio de porosidad

\begin{tabular}{|c|c|c|}
\hline Porosidad & $\begin{array}{c}\text { Sin } \\
\text { remineralizar }\end{array}$ & Remineralizada \\
\hline T22.5 & $26.63 \%$ & $31.39 \%$ \\
\hline T14.3 & $19.94 \%$ & $16.91 \%$ \\
\hline T21.4 & $30.17 \%$ & $31.48 \%$ \\
\hline $6 \mathrm{~N} 2$ & $23.93 \%$ & $30.83 \%$ \\
\hline
\end{tabular}

La dureza medida a través de la prueba Shore D del material remineralizado no mostró una mejoría evidente. Esto se debe a que las rocas presentan una gran variabilidad en su microestructura, lo cual interfiere con el procedimiento analítico que es muy puntual. Sin embargo, a nivel macroscópico fue posible notar un aumento en la resistencia del material, ya que las rocas sin tratamiento, al someterse a una prueba de caída libre, se pulverizaban o fracturaban, mientras que las rocas remineralizadas no se fracturaron.

Además tanto en las imágenes obtenidas en el MEB, como de manera macroscópica, se observó que la superficie de las rocas remineralizadas presentaban una mayor cementación de los granos de micrita y los cristales aciculares hacia el núcleo de la roca. Esto se comprobó porque que el haz de electrones no desprendió cristales, a diferencia de lo sucedido en las rocas sin tratamiento. De manera macroscópica, se observa que las rocas sin tratamiento se pulverizan y dejan residuos en las manos, mientras que las remineralizadas no lo hacen.

Con las pruebas de retención de humedad se comprobó, además, que las rocas remineralizadas presentan menor disgregación y menos pérdida de material en comparación con las rocas no tratadas, después de abruptos secados a $105^{\circ}$ y de inmersiones totales en agua durante 24 horas.

Las rocas tratadas e intemperizadas durante un año presentaron la formación de eflorescencias en la superficie. Estas sales se analizaron con DRX y se identificaron como tenardita (sulfato de sodio).

\section{Discusión de resultados}

Las rocas de la cornisa del edificio $5 \mathrm{~N} 2$ son de procedencia local, ya que corresponden a la composición mineralógica de las formaciones geológicas de las canteras cercanas a la zona arqueológica.

No hubo elección específica de la materia prima. Toda la roca de la región es más o menos de la misma calidad. Los acabados "escultóricos" se llevaron a cabo recubriendo las preformas burdas esculpidas en la roca con estuco; sin embargo, en las rocas más duras es posible notar un mejor acabado en el labrado de las figuras. 
Tabla 5. Resultados de medición de dureza Shore D.

\begin{tabular}{|c|c|c|c|c|c|c|c|c|}
\hline & \multicolumn{2}{|c|}{ T20.1 } & \multicolumn{2}{|c|}{ T21.4 } & \multicolumn{2}{|c|}{ T22.5 } & \multicolumn{2}{|c|}{$6 \mathrm{~N} 2$} \\
\hline & $\begin{array}{l}\text { Sin } \\
\text { tratamiento }\end{array}$ & $\begin{array}{l}\text { Con } \\
\text { tratamiento }\end{array}$ & $\begin{array}{l}\text { Sin } \\
\text { tratamiento }\end{array}$ & $\begin{array}{c}\text { Con } \\
\text { tratamiento }\end{array}$ & $\begin{array}{l}\text { Sin } \\
\text { tratamiento }\end{array}$ & $\begin{array}{c}\text { Con } \\
\text { tratamiento }\end{array}$ & $\begin{array}{l}\text { Sin } \\
\text { tratamiento }\end{array}$ & $\begin{array}{l}\text { Con } \\
\text { tratamiento }\end{array}$ \\
\hline & 68 & 58 & 48 & 58 & 46 & 53 & 44 & 50 \\
\hline & 70 & 45 & 50 & 48 & 44 & 54 & 52 & 51 \\
\hline & 64 & 57 & 40 & 53 & 51 & 60 & 46 & 66 \\
\hline & 58 & 53 & 53 & 57 & 43 & 64 & & 68 \\
\hline & 60 & & 63 & 54 & 46 & 64 & & 70 \\
\hline & 82 & & 67 & 48 & 50 & & & 70 \\
\hline & 58 & & 71 & & & & & 56 \\
\hline & 65 & & 63 & & & & & 50 \\
\hline & 75 & & 63 & & & & & 51 \\
\hline & 65 & & & & & & & 50 \\
\hline & & & & & & & & 65 \\
\hline Promedio & 66.5 & 53.3 & 57.6 & 53.0 & 46.7 & 59.0 & 47.3 & 58.2 \\
\hline $\begin{array}{l}\text { Desviación } \\
\text { estándar }\end{array}$ & 7.6 & 5.9 & 10.2 & 4.3 & 3.2 & 5.3 & 4.2 & 9.1 \\
\hline
\end{tabular}

Ninguna roca presenta yeso en su composición primaria. Esto refuta las hipótesis anteriores que sostenían que los sulfatos provenían de la composición de la roca. Estas sales podrían provenir del agua (de río) utilizada para la restauración o del cemento utilizado para la consolidación arquitectónica.

Los principales agentes de intemperismo son el agua y los factores biológicos. Estos agentes, que actúan mediante mecanismos químicos, poseen una estrecha relación con el contexto arqueológico en el que se encontró la cornisa y con el medio geográfico que prevalece en la región.

Existe una relación entre la clasificación realizada por observación in situ (rocas muy disgregadas, rocas disgregadas y rocas no disgregadas); y la composición, diagénesis e intemperismo de las rocas.

La remineralización genera una serie de cambios macroscópicos y microscópicos, tanto mineralógicos como físicos y químicos, que representan una optimización de las propiedades de las rocas. Esto confirma la efectividad del proceso para el tratamiento de rocas calizas disgregadas. El principal cambio que se provoca es la conversión del carbonato de calcio en fluoruro de calcio, por un mecanismo de intercambio iónico. Por eso se forman minerales pseudomorfos después del tratamiento.

Este cambio produce un mineral insoluble en la superficie de las rocas, que contrasta con la alta solubilidad del carbonato de calcio. Esto funciona como una "barrera protectora", ya que los principales mecanismos de deterioro son consecuencia de la disolución de la calcita.

El proceso de remineralización es selectivo. Las áreas donde se sustituye de mejor manera la calcita son aqueIlas conformadas por carbonatos secundarios. A éstas le siguen las zonas compuestas por micrita y, posteriormente, las conformadas por cristales de mayor tamaño. Por lo tanto, el fluoruro de sodio actúa con mayor facilidad en zonas con carbonatos neoformados que poseen una estructura inestable.

Los minerales silíceos, como arcillas y cuarzo, no son afectados por el tratamiento de remineralización.

La formación de fluoruro de calcio se lleva a cabo en la superficie de la roca. Esto se debe a los dos factores que influyen en la profundidad de penetración del fluoruro de sodio. El primero de ellos es la conformación de la superficie y de la red de poros de la roca. El segundo factor corresponde al método de aplicación del remineralizador. 
El fluoruro de sodio no actúa como una sustancia consolidante, sino como remineralizador, lo que quiere decir que transforma la composición misma de los cristales de carbonato de calcio, reconstituyendo su red cristalina, sin la precipitación de minerales en la superficie o en los poros de la roca. Además no funciona como adhesivo o cementante.

El proceso de remineralización con fluoruro de sodio cumple con los criterios teórico-normativos que rigen la praxis, considerándose como un proceso válido y legítimo. ${ }^{20}$

\section{Conclusiones}

Las rocas de la región Río Bec se ven ampliamente afectadas en su estructura por la disolución provocada por el agua ácida (ya sea en forma de lluvia, capilaridad, etcétera), así como por el metabolismo de plantas y hongos. Esto provoca cambios en la estructura de los cristales minerales que generan que la roca sea inestable y se disgregue con facilidad.

El tratamiento con fluoruro de sodio cambia la composición de las rocas calizas, generando una capa superficial porosa y con cristales minerales pseudomorfos ${ }^{21}$ a los de la roca original. Este cambio de composición provee a la roca de una capa "protectora", puesto que el fluoruro de calcio formado es insoluble en agua ácida a diferencia del carbonato de calcio de la superficie original. Además provee de cohesión a los cristales aciculares que se forman por el intemperismo de las rocas, mejorando su estabilidad y disminuyendo su grado de disgregación.

Cabe mencionar que el fluoruro de sodio no es una sustancia consolidante, ya que no actúa formando interfases de capas adhesivas como lo haría un polímero y, no actúa como una sustancia cementante como el hidróxido de calcio. Por el contrario, actúa en la composición misma de los cristales de la roca remineralizándolos, es decir reestructurando los minerales que la conforman, por eso el proceso de intervención que se realiza debe llamarse remineralización y debe estar separado de los procesos de consolidación.

\section{Referencias}

\section{Anrubio Vega, Elda Justina}

1998 "Causas y efectos de deterioro de los materiales arqueológicos calcáreos del Sur de Quintana Roo y una propuesta para su conservación", tesis de licenciatura en Restauración de Bienes Muebles, México, ENCRyM.

${ }^{20}$ Estos criterios son ampliamente discutidos y abordados en la tesis de quien escribe (Straulino 2010), en la sección 6.7 Ilamada "Parámetros de evaluación del tratamiento".

21 Cristal mineral de una composición distinta que posee la misma forma que el mineral que le dio origen.
Arnauld M. Charlotte, Dominique Michelet, Boris Vannière, Philippe Nondédéo 2008 "Houses, Emulation and Cooperation among the Río Bec Groups", presentación en el Simposio Neighborhoods in Archaeology: The Assessment of Intermediate Units of Spatial and Social Analysis, coordinado por Linda Manzanilla y M. Charlotte Arnauld, 73 Congreso Internacional de Americanistas, Vancouver, Canadá, 26-30 Marzo.

Ashurst, John, Francis G. Dimes

1998 Conservation of building and decorative stone, Oxford, Butterworth-Heinemann .

Bravo-Álvarez et al.

2003 "Efecto de la lluvia ácida a el material constituyente de los monumentos mayas mexicanos", en Pedro Martínez Pereda (ed.), Ingeniería. Investigación y tecnología, México, UNAM, vol. núm. 4, octubre-diciembre, 195-206.

Caroe, Martin

1986 "Wells Cathedral: Conservation of figure sculptures 1975-86. Final report and assessment", en Bromelle and Smith, Case studies in the conservation of stone and wall paintings, Preprint of the Contributions of the Bologna Congress, 21-26 september 1986, Londres, The International Institute for Conservation of Historic and Artistic Work.

Castro Mora, Jesús (comp.)

2002 Monografía geológico-minera del estado de Campeche, México, Consejo de Recursos Minerales, Secretaría de Economía.

Castro Barrera, María del Carmen, Martha Tapia González

1993 "Palenque. Intervenciones anteriores en conservación: seguimiento y evaluación", tesis de licenciatura en Restauración de Bienes Muebles, México, ENCRyM.

Cedillo Álvarez, Luciano

1991 "La conservación en zonas arqueológicas. Tres décadas de trabajo", tesis de licenciatura en Restauración de Bienes Muebles, México, ENCRyM.

Comisión Nacional del Agua (Conagua)

2007 Determinación de la disponibilidad de agua en el acuífero Xpujil, estado de Campeche, México, Comisión Nacional del Agua, Subdirección General Técnica, Gerencia de Aguas Subterráneas, Subgerencia de Evaluación y Modelación Hidrogeológica, documento electrónico http:// www.conagua.gob.mx/CONAGUA07/Noticias/DR_0405.pdf, consultado en septiembre de 2009.

García Solís, Claudia Araceli, Silvana Berenice

Valencia Pulido

1996 "Proyecto Chicanná, Campeche. Informe de trabajotemporada julio 1996", México, Archivo de la Coordinación Nacional de Conservación y Restauración del Patrimonio Cultural-INAH Documento impreso.

1997 "El deterioro de piedra en la zona arqueológica de Chicanná, Campeche, y una propuesta para su conservación", tesis de licenciatura en Restauración de Bienes Muebles, México, ENCRyM. 
García Vierna, Valeria, Claudia García Solís y Silvana

Valencia Pulido

1997 Informe de los trabajos de conservación realizados en la Estructura II. Diagnóstico general del sitio. Zona arqueológica de Chicanná. Campeche, octubre a noviembre de 1996, México, Archivo de la Coordinación Nacional de Conservación y Restauración del Patrimonio Cultural-INAH, texto impreso.

Lewin S.Z. y N. S. Baer

1974 "Rationale of the barium hydroxide urea treatment of decayed stone", en Studies in conservation 19, 1974, 24-35.

Michelet, Dominique et al.

2003 "Proyecto Río Bec (Campeche, Mexique). Informe de la primera temporada, del 15 de febrero al 18 de mayo de 2002", México, Consejo de Arqueología, INAH. Texto impreso.

2004 "Proyecto Río Bec (Campeche, Mexique). Informe de la segunda temporada, del 27 de enero al 25 de mayo de 2003", México, Consejo de Arqueología, INAH. Texto impreso.

2005 "Proyecto Río Bec (Campeche, Mexique). Informe de la tercera temporada, del 16 de febrero al 15 de mayo de 2004",

\section{Resumen}

Se muestran los resultados de la experimentación con fluoruro de sodio (NaF) para el tratamiento de roca caliza disgregada planteando una alternativa eficiente; las opciones hasta el momento implican una gran inversión de tiempo y recursos. Las muestras donde se aplicó NaF provienen de una cornisa de la zona arqueológica Río Bec en el municipio de Calakmul, Campeche. La composición de la roca, su deterioro y propiedades físicas fueron estudiados con diferentes técnicas analíticas (MEB, petrografía, EDS, DRX, entre otros), para compararlos con los resultados obtenidos de las muestras tratadas. Con reducida cantidad de producto y pocas aplicaciones, se logró una mejoría de propiedades físicas en las rocas tratadas y la conversión del carbonato de calcio a fluoruro de calcio, compuesto más estable ante el intemperismo. El fluoruro de sodio actúa por intercambio iónico, "remineralizando" la estructura de la roca. Se propone evaluar esta sustancia para tratamientos in situ.
México, Consejo de Arqueología, INAH. Texto impreso.

2006 "Proyecto Río Bec (Campeche, Mexique). Informe de la cuarta temporada, del 8 de febrero al 6 de mayo de 2005", México, Consejo de Arqueología, INAH. Texto impreso.

2007 "Proyecto Río Bec (Campeche, Mexique). Informe de la quinta temporada, del 5 de febrero al 4 de mayo de 2006", México, Consejo de Arqueología, INAH. Texto impreso.

2008 "Proyecto Río Bec (Campeche, Mexique). Informe de la sexta temporada, del 4 de febrero al 3 de mayo de 2007", México, Consejo de Arqueología, INAH. Texto impreso.

2009 "Proyecto Río Bec(Campeche, Mexique). Informe de la séptima temporada, 2008“, México, Consejo de Arqueología, INAH. Texto impreso.

Straulino Mainou, Luisa

2010 "Fluoruro de sodio: ¿Una alternativa para la conservación de piedra caliza disgregada a través de la remineralización? El caso de la cornisa del edificio 5N2 del Grupo A en Río Bec, Campeche", tesis de licenciatura en Restauración, México, ENCRyM.

\section{Abstract}

In this essay, results regarding the experimental use of sodium fluoride on decaying limestone rock are presented; previous treatments have required a great amount of time and resources to be invested. Samples, where the sodium fluoride was applied, are part of a cornice found at the archaeological zone of the Río Bec, in the town of Calakmul in Campeche. The composition of the rock, its deterioration and physical properties were studied by different analytical techniques (Scanning Electron Microscopy - SEM, petrography, Energy dispersive X-ray spectroscopy - EDS, X-Ray diffraction - XRD, among others), in order to compare to the samples treated. With a low quantity of sodium fluoride and few applications, an improvement of the physical properties of the rock was achieved as well as the conversion of calcium carbonate into calcium fluoride, a much more stable compound in the face of weathering. The sodium fluoride acts by ion exchange, re-mineralizing the structure of the rock. The objective of this work is to ponder upon the use of this compound for in situ treatments.

\section{Key words}

Remineralization, decaying limestone, conservation treatment, sodium fluoride.
Remineralización, caliza disgregada, tratamiento de conservación, fluoruro de sodio. 